\title{
LA ECONOMÍA DEL COMPORTAMIENTO COMO BASE PARA LA PROMOCIÓN DE ACTIVIDADES RECREATIVAS Y TURÍSTICAS
}

\author{
ANAID MENDOZA SÁNCHEZ \\ anmesa.a@gmail.com \\ ROCíO HUERTA CUERVO \\ rhuerta@ipn.mx \\ Instituto Politécnico Nacional
}

En este trabajo se exploran las posibilidades del uso de la economía del comportamiento como sustento teórico para el diseño de políticas públicas orientadas al fomento y la promoción de actividades recreativas y turísticas en el poblado de San Nicolás Tetelco, Tláhuac, para lo cual se realizó una Prueba de Control Aleatoria. Se contrastaron conceptos como la aversión a las pérdidas, el sesgo de perspectiva y de resultados, el empujón, las señales y las expectativas, para diseñar el experimento y analizar sus resultados. Se aplicó un cuestionario probabilístico para conocer las motivaciones de los pobladores, con esa base se diseñaron dos recorridos turísticos en la zona (de control y de tratamiento), en los cuales participaron 20 y 22 personas respectivamente. Los hallazgos del estudio permiten confirmar: 1) la importancia del diseño detallado de las intervenciones en grupos humanos, como medio para acercar los resultados a sus propósitos y 2) que los niveles de satisfacción mejoran cuando sus expectativas se acercan a los resultados de la experiencia, disminuyendo con ello el sesgo de retrospección.

Palabras clave: actividades turísticas, economía el comportamiento, empujón, sesgo, bienestar.

\section{BEHAVIORAL ECONOMICS AS A BASIS FOR PROMOTING RECREATION AND TOURISM ACTIVITIES}

The possibilities of using behavioral economics as theoretical support for the design of public policies are explored, aimed at the furtherance and promotion of recreational and tourist activities in the town of San Nicolás Tetelco, Tláhuac, for which purpose a Randomized Controlled Trial was performed. Concepts such as loss aversion, perspective and results bias, nudge, signals and expectations, were used to design the experiment and analyze its results. A probabilistic questionnaire was applied to find out the motivations of the inhabitants, with that base two tourist routes were designed in the area (control and study), in which 20 and 22 people respectively participated. The findings of the study confirm: 1) the importance of the detailed design of interventions in human groups, as a mean to bring the results closer to their purposes and 2) that satisfaction levels improve when their expectations are close to the experience results, thereby reducing the hindsight bias.

Keywords: touristic and recreational activities, local development, behavioral economic, nudges, bias, wellbeing. 


\section{Introducción}

L

a Ciudad de México se caracteriza por concentrar un gran número de atractivos turísticos, la mayoría de los cuales se encuentran en las alcaldías altamente urbanizadas. No obstante, los espacios rurales también albergan una amplia variedad de recursos naturales, arqueológicos, históricos y tradiciones culturales de alto valor para propios y extraños. En el catálogo de recursos turísticos de esta metrópoli se identifican 11 zonas destacadas, principalmente en las delegaciones de Xochimilco y Tláhuac, tales como espacios arqueológicos, templos coloniales, barrios antiguos, canales y chinampas, además de una variada oferta gastronómica, ejemplo de ello es la "Feria del mole" que goza de reconocimiento internacional (Sectur, 2013).

Dentro de los límites de la ciudad se localiza San Nicolás Tetelco, Tháhuac (en adelante San Nicolás), localidad que concentra en un pequeño territorio diversos recursos turísticos como una parroquia del siglo XVII, un sistema de canales y chinampas en Xochimilco que han sido declarados patrimonio cultural de la humanidad, así como una rica y variada gastronomía propia de la zona, entre otros (Sectur, 2013). Sin embargo, estos recursos no han sido aprovechados a cabalidad por los gobiernos locales para incentivar y promover actividades turísticas y recreativas, a fin de atraer a visitantes nacionales e internacionales y a la propia población residente (Mendoza, 2019).

Teniendo presentes estas características, se seleccionó el poblado de San Nicolás para realizar un ejercicio de exploración a través de una Prueba de Control Aleatoria (Randomized Controlled Trial, RCT, por sus siglas en inglés), utilizando la economía del comportamiento para el diseño de una intervención en materia de promoción turística y actividades recreativas. En dicha localidad predominan los usos y costumbres, en donde gran parte de las tierras son propiedad ejidal (Alcaldía de Tláhuac, 2020). Aunque cuentan con recursos turísticos, ni la población ni las autoridades se han ocupado de ellos por la dificultad que representa delimitar los derechos de propiedad (Mendoza, 2019; Marín, 2020).

Con estos antecedentes, el trabajo se organiza en cuatro apartados, además de la introducción y las conclusiones. En el primero se presenta el contexto de la zona de estudio; en el segundo se realiza el encuadre teórico-conceptual de la economía del comportamiento; en el tercero se detalla la metodología utilizada para el diseño del ejercicio y en un cuarto apartado se muestran los resultados y la discusión. 


\section{El contexto: San Nicolás Tetelco}

San Nicolás es una localidad que cuenta con 8 mil 997 habitantes (INEGI, 2020). De acuerdo con el Coneval (2020) su nivel de rezago es muy bajo, el $20.81 \%$ de su población mayor a 15 años tiene educación básica incompleta y el $22.92 \%$ de sus habitantes no cuenta con cobertura de servicios públicos de salud. Las principales actividades económicas del poblado son los servicios y el comercio de bienes de consumo. Las actividades de agricultura que se realizan son básicamente de autoconsumo. Actualmente, esta localidad cuenta con poco más de 200 unidades económicas y sus ingresos son de los más bajos de toda la Ciudad de México, junto con los que perciben los trabajadores de Milpa Alta (INEGI, 2020).

Es una de las localidades más antiguas del Valle de México, su existencia es anterior a la Conquista, por ello se caracteriza por poseer una vasta historia y cultura; además, cuenta con recursos naturales, pero se han ido deteriorado con el paso del tiempo ante la indiferencia de su población y las autoridades (Mendoza, 2019; Marín, 2020).

Los sitios que preservan su patrimonio se encuentran catalogados en el Inventario turístico de San Nicolás Tetelco (Mendoza, 2019) y en Lugares representativos de San Nicolás Tetelco (Marín, 2020). En esta localidad también se cuenta con patrimonio cultural intangible, como relatos y fiestas patronales, los cuales son parte de la identidad del pueblo y sus habitantes (Marín, 2020; Alcaldía de Tláhuac, 2020), aunque la unidad departamental de turismo responsable realiza acciones solo de manera esporádica (Mendoza, 2019).

La política de fomento turístico de esta demarcación se basa en sus pueblos originarios, especialmente por motivos religiosos, así como en la difusión de su gastronomía; sin embargo, esto no se materializa en San Nicolás. La desconfianza y los conflictos entre los representantes del ejido y los pobladores no les permite ni siquiera dialogar sobre las posibilidades de desarrollo de su comunidad. La información derivada de la investigación de Marín (2020) destaca la imposibilidad de analizar y canalizar posibles proyectos locales de desarrollo, ya que los representantes ejidales "se creen dueños [...] y no permiten proyectos que nos den una mejor proyección para el desarrollo en el pueblo" (entrevistado, citado por Marín, 2020, p. 100). Ante tales circunstancias, se observa la dificultad para realizar proyectos de importancia turística significativa en la zona, lo cual pudiera ser el motivo de que 
las autoridades de la demarcación hayan soslayado a San Nicolás en sus planes de promoción económica.

En comunidades en las que los usos y costumbres son muy arraigados, las condiciones que sirven de contexto para la toma de decisiones están fuertemente naturalizadas y no permiten, sin mecanismos de intermediación previamente diseñados, modificar los comportamientos asociados al sistema de valores prevaleciente. Para acercar a los habitantes a una toma de decisión que permita mejorar sus circunstancias individuales y sociales, con relación a la actividad turística y la recreación, son necesarios procesos de intervención planeada por parte de los ejecutores de políticas públicas, que amplíen las fronteras entre las cuales deliberan. Ese es el reto de las políticas públicas en el contexto local.

\section{Acercamiento teórico: la economía del comportamiento y su utilización en los procesos de toma de decisión}

La economía del comportamiento es una corriente que modifica al enfoque neoclásico en la comprensión en torno a la toma de decisiones de los individuos. A diferencia de la teoría de la utilidad esperada, la cual sostiene que una persona siempre va a elegir un paquete de consumo que maximice su utilidad, la también llamada economía conductual, retoma nociones de la psicología, la sociología y la economía para comprender con más detalle los comportamientos de las personas en procesos decisivos (Campos, 2017).

Así, la economía del comportamiento plantea la importancia de considerar las motivaciones intrínsecas y los contextos de los sujetos como medio para involucrarlos en nuevos procesos de toma de decisiones (Thaler, 2015; Kahneman y Tversky, 1979). Es decir, cambia el paradigma respecto al individuo como ser de racionalidad completa (costo-beneficio), que es capaz de hacer análisis objetivos, incluyendo cálculos complejos si se requiere, en el que las condiciones del exterior no le afectan, con el fin de obtener siempre el mayor beneficio al menor costo; adicionalmente, sostiene que las preferencias son constantes en el tiempo (Campos, 2017; Ogaki y Tanaka, 2017).

Richard Thaler (2015) denomina al individuo como "econo", invocando a la racionalidad económica que la corriente neoclásica atribuye a las personas. Sin embargo, los teóricos de la economía conductual sostienen que para comprender mejor las decisiones de los individuos se les debe considerar como seres de racionalidad aco- 
tada (Simon, 1982), que toman resoluciones sin contar con la información completa de un fenómeno y que, en ese proceso, sus motivaciones internas como expectativas, sesgos, aversiones, entre otros aspectos, influyen en sus decisiones, lo cual les permite dirigirse a mejores opciones si modifican sus percepciones sobre un fenómeno en particular.

En ese sentido, es posible afirmar que las elecciones de los individuos, al no responder únicamente a incentivos económicos, pueden variar en el tiempo e incluso ser contrastantes (Kahneman y Tversky, 1979; Thaler, 2015; Campos, 2017; Ogaki y Tanaka, 2017). Para los teóricos de la economía del comportamiento "las personas no son ni enteramente racionales, ni enteramente egoístas y sus gustos son todo, menos enteramente estables" (Kahneman, 2013, p. 445).

Las nociones que introduce la economía conductual permiten elaborar planteamientos más completos al predecir la decisión final de los individuos ante determinadas opciones o situaciones. En primer lugar, Kahneman (2013) y Thaler (2015) explican que la mente humana responde acorde con dos sistemas: el primero de ellos condicionado por el temperamento, la formación familiar y los estados de ánimo que lleva a las personas a dar respuestas rápidas frente a los eventos que enfrenta: "opera de manera rápida y automática, con poco o ningún esfuerzo y sin sensación de control voluntario" (Kahneman, 2013, p. 32). El segundo sistema reclama concentración, reflexión de la respuesta y control frente a la misma: "centra la atención en las actividades mentales esforzadas que lo demandan, incluidos los cálculos complejos. Las operaciones de este sistema están a menudo asociadas a la experiencia subjetiva de actuar, elegir y concentrarse" (Kahneman, 2013, p. 32).

Con este planteamiento, la economía del comportamiento rompe de manera significativa con la economía neoclásica, ya que considera que en los procesos de toma de decisión no solo interviene la racionalidad de los actores. Dentro de la teoría conductual, Kahneman (2013) explica los conceptos de perspectivas o prospectos como una parte sustantiva de su teoría.

Partiendo de una crítica a los planteamientos de la teoría de la utilidad de Bernoulli, Kahneman (2013)1 y Thaler (2015) demostraron que para la toma de una decisión no basta con conocer la ganancia o pérdida absoluta que generará, sino el punto de

1 Aunque en el libro Thinking fast and slow aparece exclusivamente Kahneman como autor, él mismo a lo largo del libro explica el trabajo conjunto realizado con Amos Tversky durante muchos años y que dio como resultado las aportaciones que ambos han hecho a la economía del comportamiento. 
partida previo a ella. Incorporó el efecto de la certidumbre o incertidumbre en los procesos de resolución, los puntos de referencia o de partida para evaluar un posible resultado, la aversión a la pérdida como una respuesta ante opciones probables, la sensibilidad decreciente y la ponderación de la elección.

El "efecto certidumbre" es la preferencia por alternativas con una probabilidad mayor por encima de la utilidad total (Kahneman y Tversky, 1979). Los puntos de referencia son las condiciones y los conocimientos previos que los individuos toman en consideración para evaluar posibles ganancias o pérdidas de una decisión. La conclusión de que el punto de referencia es fundamental para la forma en que las personas evalúan los resultados de sus elecciones, introdujo en Kahneman (2013, p. 470) la necesidad de estudiar la aversión frente a posibles pérdidas: "las personas siempre desean ganar y es más fuerte su aversión a la pérdida que la esperanza de una posibilidad de ganancia" .

Cuando las pérdidas se valoran como pequeñas, en función de la riqueza total de las personas, puede presentarse una disminución de la sensibilidad ante dichas pérdidas. Sintetizando su crítica a la teoría de Bernoulli (de la utilidad esperada), que la economía neoclásica retomó plenamente, Kahneman (2013, p. 475) concluyó: "los humanos que describe la teoría de las perspectivas actúan movidos por el impacto emocional inmediato de las ganancias y las pérdidas, sin pensar en perspectivas a largo plazo de riqueza y utilidad global".

Adicionalmente, la teoría de la economía del comportamiento elabora proposiciones que se involucran con las políticas públicas, como la "arquitectura de decisión". Thaler y Sunstein (2008) definen a un arquitecto de decisión como quien indirectamente influye en las elecciones de otras personas; el gobierno de cualquier escala, al establecer políticas o programas, instaura o enmarca el contexto en el que los individuos toman una elección, por lo que, en definitiva, es un arquitecto de decisión que puede tornarse preponderante (Thaler y Sunstein, 2008).

El Paternalismo libertario se refiere directamente al modo en el que se concibe la elaboración de la política pública. Cuando se utiliza el término libertario para modificar la palabra paternalismo, se hace referencia simplemente a una libertad preservada (Thaler y Sunstein, 2008). Se trata de hacer fácil para la gente seguir su propio camino ofreciéndole las mejores opciones, de manera amigable y de fácil adopción; no sobrecargar a quienes ejercen su libertad y de este modo hacerlos mejores selectores, de acuerdo con su propio juicio (Thaler y Sunstein, 2008). 
Otros conceptos centrales en la economía del comportamiento son los heurísticos: los sesgos de retrospección, las expectativas, los empujones (nudges), las señales y las opciones predeterminadas (defaults). Los heurísticos son atajos que utiliza el cerebro para operar de manera más eficiente su recurso más escaso: la atención. Ayudan al cerebro a no tener que profundizar en el contexto de una decisión, sino simplemente a identificarla como buena para él y realizar una acción. Los heurísticos en ocasiones son vulnerables a errores de percepción, valoración y juicio (Pfarr, 2016).

En economía del comportamiento es común utilizar los heurísticos de representatividad, disponibilidad, anclaje y ajuste en las RCT. El de representatividad se utiliza para juzgar con base en lo que se parece; se refiere a los estereotipos, los cuales al generar moldes estimulan una respuesta, la cual puede estar muy alejada de la realidad (Thaler y Sunstein, 2008).

Los heurísticos de disponibilidad tienen que ver con la memoria y aquella información que nos remite a asociar un evento o pregunta a datos almacenados previamente en la memoria y que no necesariamente son fieles a la realidad. Los patrones o políticas de comunicación hacia la sociedad tienen sesgos y ellos influyen en la disponibilidad de información que el cerebro va a utilizar. Anclaje y ajuste se refieren a dar puntos de referencia, a relacionar eventos con cantidades que actúan como anclas a la hora de tomar decisiones (Thaler y Sunstein, 2008); por ejemplo, se tienen nociones sobre los precios válidos o justos de determinados productos, lo cual lleva a reaccionar cuando una nueva cantidad muy alejada a la anclada se presenta en un proceso de decisión, pero en ello la mente opera con ajustes.

Los sesgos de retrospección se refieren a valorar los resultados más por las expectativas que se tienen en torno a un evento que por las posibilidades reales del mismo. Este sesgo es especialmente importante a la hora de diseñar y anunciar programas públicos, significativos para la población. Si las expectativas que se generan en torno a un programa o política son evidentemente diferentes y superiores a los resultados que se presentan, los niveles de insatisfacción serán mayores a si la política hubiese sido anunciada con sus posibilidades reales. La insatisfacción es una fuente de desdicha para las personas y por tanto de sus niveles de bienestar. Entre peores son los resultados, mayor es el sesgo de retrospección (Thaler y Sunstein, 2008).

El "empujón" o nudge es "cualquier aspecto de la arquitectura de elección que altera el comportamiento de las personas de una manera predecible, sin prohibir ninguna opción o cambiar significativamente sus incentivos económicos" (Thaler y Sunstein, 
2008 , p. 6). Los autores explican que para poder considerarse como un empujón (aspecto que altera el comportamiento) debe ser fácil y barato de adoptarse o evitarse; un mandato o una prohibición no pueden ser considerados como nudges, ya que no son aceptados voluntariamente por el actor.

Las opciones predeterminadas, como su nombre lo indica, son alternativas elaboradas que se presentan para dirigir la decisión hacia cierta opción deseable. Por ejemplo, si en un cuestionario de salud en lugar de expresar "si desea ser donador marque esta casilla", se cambia la opción a "si no desea ser donador marque esta casilla", se está "empujando" a que todos sean donadores, pero si alguien no está dispuesto puede señalarlo con una sencilla marca. Estas opciones pueden actuar como potentes empujones (Thaler y Sunstein, 2008), que si bien no representan un compromiso inmediato exhiben una elección que, en muchos casos, mueve a las personas de su valoración previa.

En el caso del turismo o las actividades recreativas, las decisiones predeterminadas son utilizadas en ciertas promociones en donde se exponen dos ofertas o más y garantizan un estímulo real para que se opte por consumir inmediatamente. La aplicación de esta corriente en la formulación de la política pública ha sido exitosa en diferentes áreas como salud, ahorros para el retiro y recaudación de impuestos, por mencionar algunas; su uso continúa explorándose en distintas áreas como el turismo, visible en los trabajos de Mosalev, (2020), Wallstam et al. (2020), Gursoy et al. (2020) y Cati (2020).

Los instrumentos de la economía conductual se caracterizan por ser de bajo o nulo costo y por no imponer una carga a los contribuyentes (Thaler y Sunstein, 2008). Si la incorporación de esta corriente permite observar cambios positivos en diferentes aspectos de la vida pública, sin incrementar de manera relevante el gasto gubernamental, es pertinente considerar su aplicación.

En el caso de San Nicolás, donde hay una fractura evidente entre los miembros de su comunidad que impide el desarrollo de proyectos locales de mayor envergadura que mejoren su bienestar, la utilidad de programas orientados por la economía del comportamiento puede ser mayúscula, ya que ayudarían a romper círculos viciosos que alientan el inmovilismo, la apatía y el estancamiento de la localidad y su comunidad. Esta teoría se apoya frecuentemente en experimentos y a través de su aplicación es posible observar si las nociones teóricas propuestas generan los comportamientos esperados, estas pruebas se utilizan para observar los efectos de las 
intervenciones en experimentos sociales, de manera que sirven para corroborar el funcionamiento de una política (Haynes et al., 2012).

\subsection{La economía conductual en el turismo y la recreación}

Si bien las políticas de promoción privada del turismo utilizan mensajes especiales para motivar a las personas a consumir ciertos productos turísticos y dichas políticas están influidas por conceptos que buscan incidir en las percepciones y emociones de las personas, la mayoría de las investigaciones en turismo reportan estudios transversales que "solo exhiben correlaciones o relaciones predictivas entre variables, pero no relaciones causales" (Gursoy et al., 2020, p. 707). Para estos autores los estudios transversales "ha(n) sido el método predominante en la investigación turística" y "el diseño experimental ... sigue siendo relativamente infrautilizado en el estudio de los fenómenos relacionados con el turismo" (Gursoy et al., 2020, p. 707).

Mosalev (2020) empleó la teoría del comportamiento en estudios transversales e identificó diversos factores que afectan las decisiones de los turistas y que no están determinados por la industria del turismo, como la liberalización de los trámites turísticos en los países, las asociaciones estratégicas entre naciones, el terrorismo y la inseguridad; también aquellas variables que en diversos aspectos dependen de la acción de las agencias o empresas del ramo como la gestión de los flujos turísticos, cerrando con elementos del tipo descuentos y reservas sin condicionamiento.

Cati $(2020$, p. 2) analiza tres aspectos característicos de la industria turística a partir del lente conceptual de la economía del comportamiento: primero, el sesgo de aversión a las pérdidas que "puede afectar la toma de decisiones en el sector turístico, ya que los turistas tienden a poner más énfasis en lo que puede salir mal y prefieren la opción predeterminada en lugar de arriesgar un poco". En segundo lugar, analiza el sesgo de percepción de escala a partir de encuestas de satisfacción aplicadas a los turistas; finalmente, el enfoque de empuje con mensajes que buscan limitar el deterioro ecológico de los lugares turísticos.

Wallstam et al. (2020, p. 123) demuestran que "las evaluaciones económicas ex post han dominado el discurso de evaluación" en el turismo... "Ios marcos utilizados para decidir el mérito... se han centrado abrumadoramente en parámetros económicos", incluso en los programas gubernamentales. Gursoy et al. (2020) identificaron seis artículos específicos que hicieron uso de la economía del comportamiento en estudios realizados entre 2010 y 2019. Dichos estudios evaluaron comportamientos 
y experiencias de los consumidores en sus recorridos turísticos; la disonancia cognitiva de las promociones ante escasez de recursos; la excitación individual ante el "turismo virtual"; la satisfacción de los clientes ante servicios prestados por personas con alguna discapacidad; la predilección por menús impresos o mostrados en tabletas y las preferencias ante una atención robotizada a la tradicional.

Los trabajos referidos líneas arriba enfatizan las aportaciones de la economía del comportamiento en los sesgos que se construyen entre turistas y prestadores de servicios, a partir de las expectativas y los resultados de sus experiencias, en relación con resultados longitudinales de otras investigaciones.

Estudios como el planteado en este trabajo no fueron identificados en la búsqueda bibliográfica. Su aportación puede ser significativa, especialmente para los gobiernos locales que buscan planear y diseñar políticas y programas dirigidas a promover la recreación turística en sus territorios y el uso del tiempo de ocio entre sus habitantes.

\section{Metodología: diseño del experimento}

El diseño metodológico parte de un estudio de caso, a través de una estrategia mixta que identificó las áreas de oportunidad para la construcción de empujones que incentivaran la oferta y la demanda de actividades de recreación y turismo. La estrategia de investigación incluyó una RCT para evaluar el impacto de los nudges aplicados en el comportamiento de los potenciales consumidores (Duflo y Banerjee, 2011).

De tal manera, el estudio de caso fue la estrategia de investigación utilizada, ya que los RCT implican la delimitación precisa de la población a intervenir, a partir de la identificación de un problema concreto. Para Duflo y Banerjee (2011, p. 133) "Ios empujones pueden ser especialmente útiles cuando, por cualquier razón, los hogares dudan sobre los beneficios de lo que se propone para ellos. Esto hace que la atención preventiva sea un candidato doblemente apropiado para tales políticas". En el combate a la pobreza, los RCT que han predominado han sido precisamente diseños experimentales enfocados a poblaciones delimitadas por un problema concreto. En su libro Poor economics, Duflo y Banerjee (2011) exponen un considerable número de casos con RCT a partir de las problemáticas y condiciones específicas de las comunidades. 
En este trabajo las características del caso seleccionado fueron: contar con servicios urbanos, con recintos para el turismo cultural, histórico, religioso y alternativo; esta información se obtuvo del programa de trabajo de la localidad estudiada (JUD de Fomento a la Actividad Turística, 2018). Los atributos descritos son análogos a los de otros pueblos de la Alcaldía de Tláhuac, como San Juan Ixtayopan y San Pedro Tláhuac; sin embargo, a diferencia de esas localidades, en San Nicolás la actividad relacionada con el turismo y la recreación es nula, a pesar de los recursos con que cuenta.

El diseño de la prueba en campo o RCT se apoyó de distintas estrategias de recogida de información referidas a continuación: la observación participante permitió identificar a los actores clave, la dinámica económica, las características del tejido social, los recursos con los que cuenta la comunidad y las actividades en materia de turismo y recreación; mediante la revisión de documentos oficiales se conoció más a fondo el patrimonio con el que cuenta la Alcaldía, especialmente el caso de San Nicolás y el calendario de festividades. También se revisó el programa de trabajo de la unidad de promoción turística (JUD de Fomento a la Actividad Turística, 2018).

A través de entrevistas presenciales semiestructuradas a actores clave (ejidatarios, coordinador territorial, integrantes del grupo chinampero, locatarios, historiador del pueblo, participantes del colectivo Tetelcóatl, así como diferentes habitantes del pueblo) se estudió con profundidad la dinámica económica, social y la relación de la comunidad con las autoridades locales. La entrevista estructurada a los responsables locales en materia de turismo permitió conocer la implementación del programa de trabajo del área y la relación con la comunidad.

Otra técnica cualitativa empleada fue la reunión de un grupo de enfoque con los actores clave de la comunidad de San Nicolás, con el objetivo de identificar los sitios de interés susceptibles de ser integrados a una ruta turística, la justificación de su elección, las actividades a realizar y la información que debía considerarse para los recorridos en la RCT, es decir, la oferta de turismo. El conjunto de actividades descritas incorporó la participación deliberativa de un segmento de la población.

A partir de la sistematización de la información recabada, se generaron las categorías de análisis para identificar los elementos en la toma de decisión de los individuos con respecto de las actividades turísticas y recreativas. Estas categorías fueron llevadas a un cuestionario estructurado, aplicado en campo, el cual estaba dirigido a personas mayores de edad, habitantes de la comunidad estudiada. 
El tipo de muestra es aleatoria simple, no probabilística. El cuestionario se aplicó a 104 personas mayores de edad residentes en San Nicolás, los días 31 de marzo, 6, 7 y 13 de abril del 2019. Se elaboró una base de datos con la información recabada, se tabularon los eventos significativos y se procedió al análisis estadístico descriptivo.

El cuestionario permitió conocer los elementos en la toma de decisión con relación a las actividades recreativas en la comunidad. Esta información posibilitó identificar cuáles son las preferencias actuales de la población para este tipo de actividades, sus motivaciones intrínsecas, cómo conciben el contexto de su localidad, a qué tipo de instituciones se apegan, en qué tipo de organizaciones formales e informales se desenvuelven, así como la determinación de los recursos con los que se cuenta.

Adicionalmente, se elaboró un diagnóstico de la comunidad, reforzado con datos obtenidos del Instituto Nacional de Estadística y Geografía (INEGI) y el Directorio Estadístico Nacional de Unidades Económicas (DENUE). Para dicho diagnóstico, se realizaron diferentes mapas del polígono urbano del poblado, ubicando así los elementos que conforman a la comunidad dentro del territorio; de este modo fue posible interpretar a profundidad el caso de estudio.

Se tomó como base la propuesta de Richburg-Hayes et al. (2014) para generar una $\mathrm{RCT}$, bajo el esquema que se presenta en la figura 1. La definición se entiende como la identificación del problema de interés. Por diagnóstico se refiere a reunir datos para crear un proceso, formar una ruta, reconocer los puntos de entrega e hipotetizar los cuellos de botella. El diseño consiste en proponer una lluvia de ideas basada en el comportamiento de los individuos (los habitantes de San Nicolás), para registrar las situaciones críticas. Finalmente, la prueba consiste en analizar las intervenciones conductuales a través de una asignación aleatoria u otra estrategia. La descripción a detalle de la RCT se muestra en el apartado 5 de este trabajo, denominado Resultados y discusión. Cabe señalar que para la ejecución de la prueba se logró la colaboración de vecinos y autoridades locales. 
Figura 1. Fases para el diseño de una intervención desde la economía del comportamiento

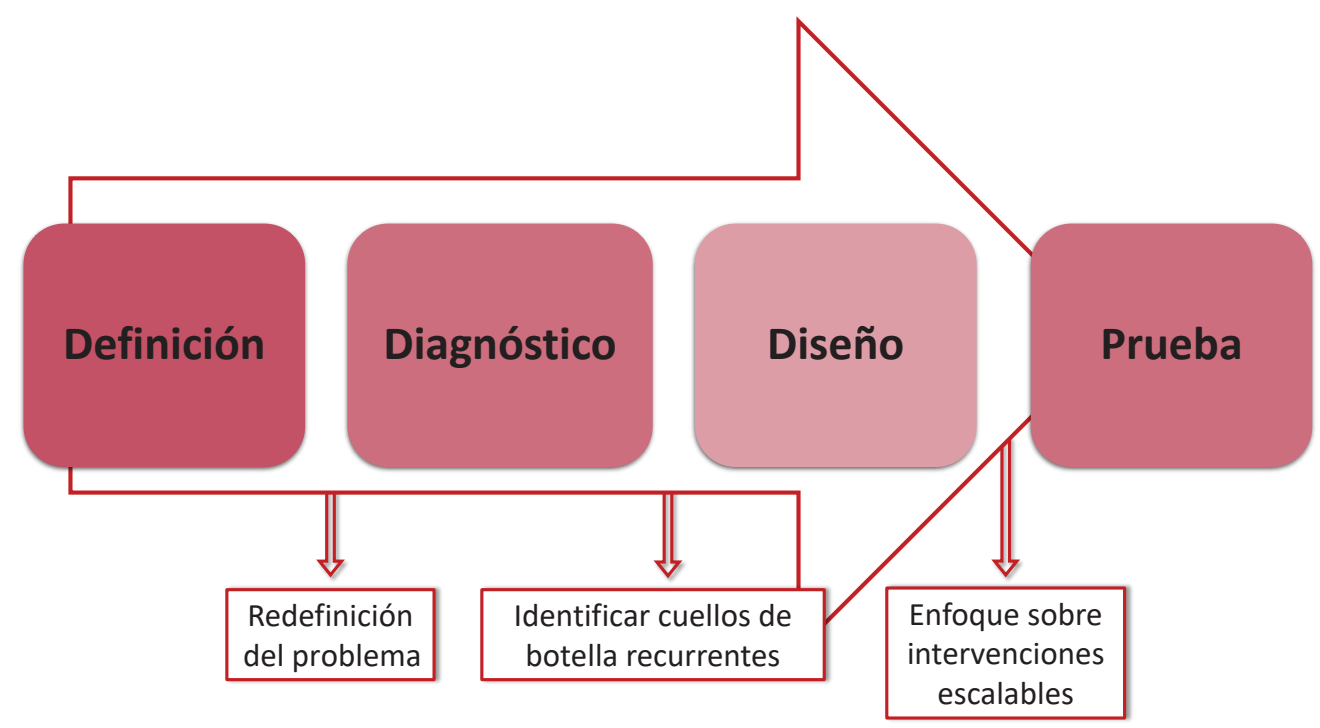

Fuente: Elaboración propia con base en Richburg-Hayes et al. (2014).

Los espacios locales son ideales para observar la efectividad de las aplicaciones teóricas-prácticas de la economía del comportamiento. Este mismo acotamiento territorial resulta el óptimo para la planeación, organización y gestión de la actividad turística de manera más favorable, ya que es en este espacio en el que interactúan la oferta, la demanda y la población residente (Wallingre, 2014).

\section{Resultados y discusión: la ejecución de la Prueba de Control Aleatoria}

Mediante las derivaciones obtenidas con los diferentes instrumentos y herramientas metodológicas, se procedió a realizar una triangulación entre la información documental y la empírica. Esta información fue la base del diseño de la RCT; en este tipo de pruebas se emplean dos grupos, uno de control y el otro de tratamiento, los grupos se comparan antes y después de realizar la prueba para analizar los resultados del tratamiento sobre el segundo grupo y así contrastar la eficiencia de las nociones teóricas empleadas.

Como se mencionó anteriormente, las actividades previas permitieron que junto con los pobladores se elaborara un producto turístico, el cual consistió en una ruta por la comunidad. Para efectos del diseño de la RCT, el recorrido se realizó en dos 
ocasiones, el primero de control y el segundo de tratamiento, en las fechas 28 de abril y 5 de mayo de 2019 respectivamente, realizados en domingo y con una semana de diferencia. El propósito de realizarlos en el mismo día de la semana fue lograr que la dinámica predominante en la ruta fuera básicamente la misma. Las condiciones fueron iguales: los lugares a visitar, la información proporcionada y las actividades recreativas que se realizaron, es decir, la oferta.

En ambos el tiempo previo de difusión fue de tres días; el primer punto de reunión fue el centro de la Alcaldía, lugar en el que se ofreció un transporte que llevaría al punto de partida (la curva). La ruta tuvo una duración de tres horas y se muestra en la figura 2.

Figura 2. Mapa de la ruta realizada

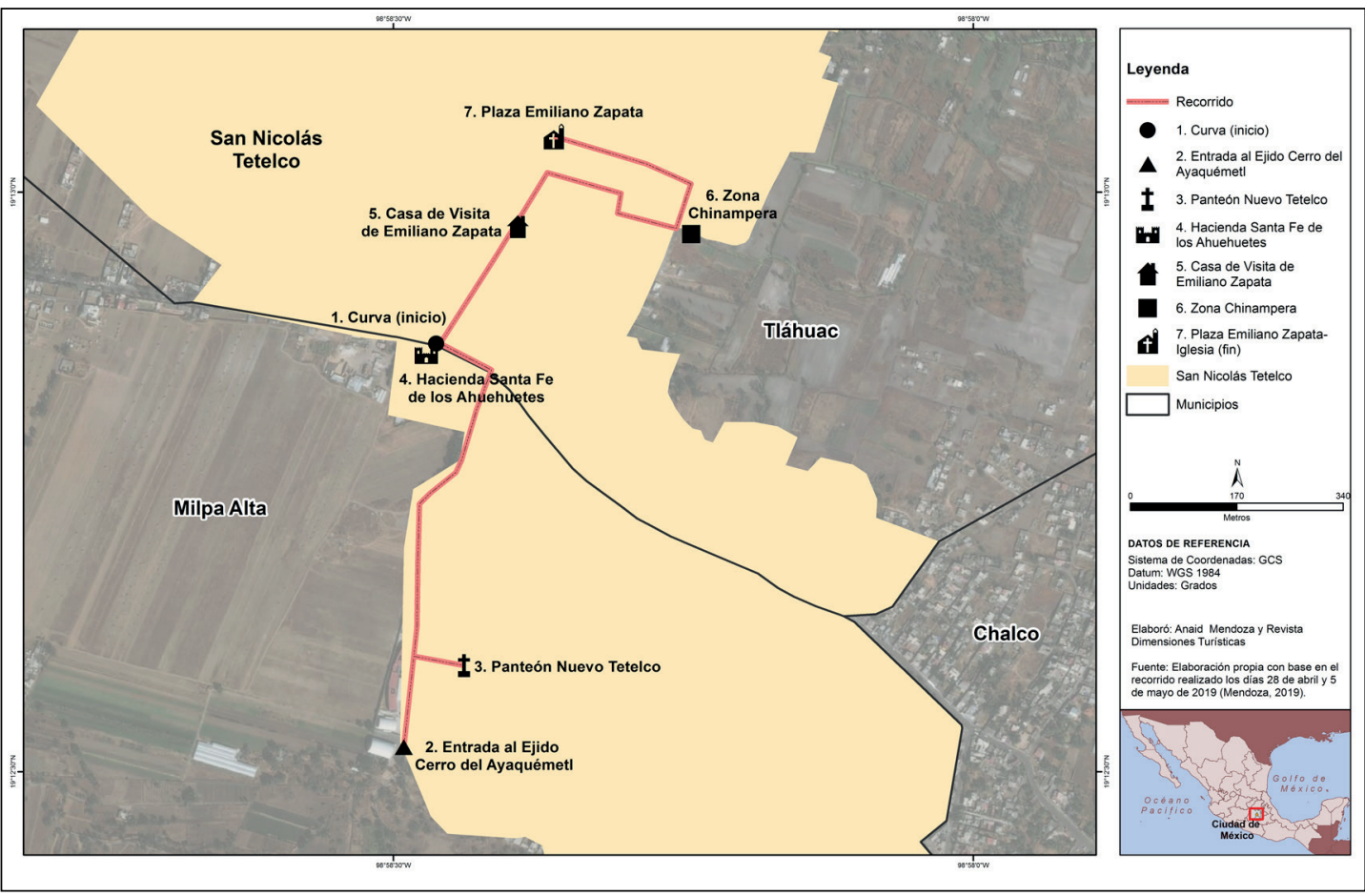

Fuente: Ver interior figura 2.

Se incorporaron señales en el recorrido de tratamiento, mientras que el de control se difundió mediante la estrategia tradicional de la autoridad territorial (coordinación), que consiste en invitar a la población a través de carteles elaborados a mano, los cuales se colocan en diferentes puntos del pueblo. La información en los carteles incluye nombre, día, hora, lugar y algunas especificaciones generales, siendo 
muy básicos y sin imágenes. De manera adicional, la coordinación territorial realizó un cartel digital, el cual publicó en su red social de Facebook; si bien ese cartel sí contenía imágenes, la información proporcionada era básicamente la misma.

Las señales introducidas en la difusión de los mensajes fueron la única diferencia que existió entre el recorrido de control y el de tratamiento. Mediante la promoción, se enviaron las señales a través de mensajes, los cuales hicieron referencia a diferentes aspectos de interés identificados a través del cuestionario y elementos que se dieron para incidir en la toma de decisión de los individuos con respecto a la realización de actividades turísticas y recreativas. Estos puntos de referencia hicieron énfasis en lo que la población reconoció como importante: la naturaleza, la cultura y la historia.

Con respecto a las motivaciones intrínsecas, se encontró que más del $90 \%$ de la población considera importantes los recursos y la belleza natural, mientras que más del $80 \%$ observan como relevantes la cultura y la historia. A través de la encuesta se pudo apreciar que los recursos naturales fueron el elemento más valorado por los participantes. Esta información fue utilizada en la difusión con tratamiento.

Los heurísticos incorporados en los mensajes fueron los de representatividad, anclaje y ajuste. En los carteles con tratamiento se mostraron fotografías de los atractivos considerados en el poblado, de manera que quienes los apreciaran fueran capaces de evocar en su mente otros sitios similares. El anclaje se realizó proporcionando el precio de una ruta con las mismas características, en algún lugar semejante, resaltando a la par el concepto gratuito de este recorrido. El de ajuste se utilizó limitando el número de personas; además, se expresó que la actividad sería por única ocasión, haciendo notar que era una oportunidad y de esta manera aludir a la aversión a las pérdidas. A través de los mensajes también se hizo alusión al efecto IKEA (Norton et al., 2011), el cual se refiere a que las personas valoran más las actividades en las que tienen un papel activo que aquellas en las que no participan.

El empujón incorporado para facilitar la acción fue el transporte, mismo que se proporcionó desde el centro de la Alcaldía hacia el sitio seleccionado. Los mensajes elaborados para el recorrido con tratamiento fueron claros con respecto a que en el poblado existen sitios de interés por conocer y actividades por realizar; de esta manera se proporcionó certeza, eliminando la aversión a las pérdidas y empujando de esta forma a la acción. El diseño de estos mensajes fue sencillo, claro, innovador y honesto, características que disminuyen la sobrecarga para los individuos, lo que 
les permite realizar una evaluación rápida de la opción que se les presenta y simplificar el proceso de decisión.

Los carteles con el tratamiento fueron utilizados de manera digital por la Alcaldía en sus redes sociales. Adicionalmente, la coordinación territorial hizo uso de ellos de manera física, colocando lonas de $3 \times 2$ metros en los puntos de entrada y salida del poblado, un paso obligado para la gente que se traslada a las afueras de la comunidad o cruza por esta para llegar a su destino. Mientras más puntos de referencia o señales, la posibilidad de que sean recibidas es mayor.

Para realizar la posprueba a través de evidencia se aplicaron dos cuestionarios, uno al inicio y el otro al final de la ruta. Estos cuestionarios hicieron posible analizar los resultados obtenidos diferenciando el grupo de control del grupo con tratamiento. El primero fue básicamente un registro de asistentes, el cual permitió conocer si la persona era un excursionista residente del pueblo o no. Adicionalmente, se preguntó ¿cómo se enteró? y ¿qué lo motivó a asistir? Estas cuestiones se establecieron para identificar cómo funcionó la difusión en cada recorrido.

El segundo cuestionario, aplicado al final de cada recorrido, pretendía obtener información sobre cómo funcionaron los mensajes enviados, las expectativas y la satisfacción de los excursionistas, para lo cual se plantearon las siguientes preguntas: ¿el recorrido cumplió con sus expectativas?, ¿recomendaría el recorrido?, ¿cambió su percepción de San Nicolás?, ¿mejoró o empeoró?, ¿qué le gustó más y qué menos?, ¿le gustaría que los recorridos continuarán? Cabe destacar que este instrumento fue el insumo para el análisis final de la posprueba de la RCT.

Los resultados de la RCT se analizaron con base en las respuestas de los cuestionarios aplicados de manera previa y al final de cada recorrido. El primero (28 de abril de 2019) fue de control y se contó con una asistencia de 20 personas, incluyendo al personal de la Alcaldía. Esta cantidad rebasó las expectativas debido a que frecuentemente este tipo de actividades carecen de apoyo y concurrencia de la población. Los resultados se pueden observar en la siguiente tabla. 
Tabla 1. Resultados de la Prueba de Control Aleatoria RCT

\begin{tabular}{|c|c|c|}
\hline Variables & Recorrido de control & Recorrido con tratamiento \\
\hline Residente de San Nicolás & $80 \%$ & $59 \%$ \\
\hline $\begin{array}{c}\text { Medio por el que se enteró del recorrido } \\
\text { Facebook } \\
\text { Por un conocido }\end{array}$ & $\begin{array}{c}5 \% \\
95 \%\end{array}$ & $\begin{array}{l}45 \% \\
55 \%\end{array}$ \\
\hline \multicolumn{3}{|l|}{ ¿Qué lo motivó a asistir? } \\
\hline $\begin{array}{l}\text { Invitación de un conocido } \\
\text { Gusto por los recorridos } \\
\text { Interés por el lugar }\end{array}$ & $\begin{array}{l}85 \% \\
10 \% \\
---\end{array}$ & $\begin{array}{c}28 \% \\
--- \\
59 \%\end{array}$ \\
\hline Cumplió con sus expectativas (Sí) & $80 \%$ & $100 \%$ \\
\hline Lo recomendaría (Sí) & $80 \%$ & $100 \%$ \\
\hline Cambió su percepción sobre San Nicolás (Sí) & $80 \%$ & $92 \%$ \\
\hline Mejoró su percepción sobre San Nicolás (Sí) & $80 \%$ & $73 \%$ \\
\hline Le gustaría que continúen (Sí) & $90 \%$ & $100 \%$ \\
\hline
\end{tabular}

Entre los principales resultados que arrojaron ambas encuestas destacan los siguientes: los excursionistas en su mayoría fueron locales ( $80 \%$ y $59 \%$ ); en ambos casos sobresale el hecho de que los asistentes se enteraron del recorrido por medio de algún conocido (95\% y 55\%) y en menor media por Facebook (5\% y $45 \%$ ), esto indica que se mantuvieron los canales tradicionales de comunicación.

El $85 \%$ de los encuestados del primer recorrido manifestó que el motivo para asistir fue porque un conocido lo invitó, mientras que en el segundo el 59\% indicó como motivación su interés por el lugar. Como se puede observar en la tabla 1, más de tres cuartas partes de los participantes en ambos circuitos mencionó que el recorrido sí cumplió con sus expectativas, que sí lo recomendarían y que a partir de esta experiencia sí cambió su percepción sobre el poblado y en la mayoría de los casos mejoró y, finalmente, dijeron que sería deseable continuar con estas actividades ( $90 \%$ y $100 \%)$, lo que permite observar que se lograron establecer puntos de referencia positivos para continuar con el desarrollo de acciones turísticas, lo que permitiría incorporarlas en la agenda local (ver tabla 1).

De acuerdo con los resultados, se puede advertir que las expectativas generadas por los anuncios de los carteles se correspondieron en general con lo vivido a lo largo del recorrido. Kahneman (2013) se refiere al sesgo de retrospección cuando una decisión se valora por los resultados que dicha elección generó y no por las opcio- 
nes que objetivamente se tenían. Cuando los resultados se alejan de lo imaginado y deseado, este sesgo de retrospección crece. Se califica como errónea la decisión cuando el resultado obtenido no corresponde con lo previsto o imaginado.

Las valoraciones sobre las posibilidades reales de las opciones que se tenían se desdibujan, ya que el peso del resultado define la valoración. En este caso y teniendo presente los resultados que arrojaron los cuestionarios, es posible inferir que el sesgo de retrospección fue mínimo, ya que la mayoría calificó como satisfactoria su experiencia y el único aspecto que alejó lo imaginado de lo vivido fue la basura encontrada en las chinampas.

Para los organizadores del recorrido el sesgo de retrospección también fue bajo, ya que la respuesta de los participantes se ajustó a las expectativas y supuestos formados cuando diseñaron el experimento. En ese sentido, la decisión de los organizadores de que los carteles no prometieran más de lo que realmente se podía ofrecer, puede calificarse como positiva.

Al ser esta una actividad gratuita, podría suponerse que la valoración de los participantes necesariamente debe ser positiva, pero no siempre es así. Las personas valoran también la forma en cómo emplean su tiempo y, en este caso, al concluir el recorrido reforzaron el supuesto de que participar en el mismo fue una disposición adecuada. No hubo sentimiento de pérdida. El empujón generado por el transporte gratuito motivó a quienes tenían interés por conocer el lugar y que no eran de San Nicolás, ya que en la segunda ruta de estudio el número de participantes no residentes del poblado aumentó.

En virtud de que ambos recorridos fueron iguales y que las diferencias incorporadas fueron el diseño de los mensajes y la manera de ofrecer el transporte gratuito, se deduce que las señales y el empujón fueron los elementos que marcaron la diferencia en el porcentaje de satisfacción entre ambos recorridos; no obstante, el hecho de que $41 \%$ de los participantes hayan sido visitantes externos al poblado, a diferencia del $20 \%$ con esta característica en el recorrido de control, pudo haber incidido también en los resultados de satisfacción, ya que los no residentes de San Nicolás experimentaron algo nuevo y agradable. Las novedades pueden generar sorpresas, "la dotación de bienes nuevos y satisfactorios" amplía los márgenes de utilidad y aunque el bienestar que generan sea momentáneo y pasajero, inciden en la percepción que de ellos se forma, ya que son "experiencias ilusionantes" (Kahneman, 2013). 
Más del 50\% de los excursionistas manifestó que su motivación estuvo relacionada con su interés por conocer el lugar, la cultura y/o la historia. Se confirma que los incentivos no solo fueron externos en la toma de decisiones, sino también internos (Kahneman y Tversky, 1979; Campos, 2017; Ogaki y Tanaka, 2017). Igualmente, a través de este trabajo se constató que este tipo de elementos puede aprovecharse para impulsar el diseño de políticas y programas turísticos. La no aversión a las pérdidas puede asociarse a la información precisa que se brindó, en la cual se anunciaron explícitamente los tiempos del recorrido, se ofrecieron imágenes de los lugares y un empujón.

Este hallazgo es de suma importancia porque, como lo asevera Kahneman (2013), reafirma la importancia de dar certeza de lo que obtendrán a quienes participan en una experiencia determinada, aún más cuando la experiencia es desconocida. Asumir en el diseño de los programas la aversión a las pérdidas (que los humanos recurrentemente manifestamos), así como el sesgo de retrospección, es indispensable para proyectar sus posibles resultados de una mejor manera.

A través de las señales, los empujones y el diseño, se estimuló a los visitantes a valorar objetivamente el tiempo que dedicarían en el recorrido y a identificar lo que obtendrían de esa experiencia. La decisión tomada de asignar su recurso (el tiempo) en la ruta propuesta, se vio reforzada por los resultados que se obtuvieron. En este sentido, se observó que el bienestar logrado a partir de la actividad estuvo asociado a las experiencias subjetivas de los participantes, más allá de los recursos económicos que pudiesen haber empleado, tal como lo señalan Kahneman y Tversky (1979), Campos (2017) y Ogaki y Tanaka (2017).

Las respuestas de los participantes pueden ser un buen indicador para fomentar el desarrollo de actividades de recreación y turismo por parte las autoridades competentes, ya que pueden fungir como un antecedente confiable para su futura utilización en el diseño de programas públicos en esta área.

\section{Conclusiones}

A través de este trabajo es posible afirmar que las nociones teóricas y las aplicaciones de la economía del comportamiento, empleadas de manera sostenida y sistemática en el campo del turismo y las actividades recreativas, podrían obtener resultados positivos con respecto de la toma de decisión esperada de los individuos. El espacio local es idóneo tanto para la aplicación de RCT, como para la planeación y 
organización de acciones del sector turístico y afines, ya que es posible conocer los recursos, motivaciones intrínsecas, el contexto, las instituciones y organizaciones formales e informales que imperen en el sitio.

Los recursos no económicos como el tiempo y el conocimiento, así como las motivaciones intrínsecas y el contexto, son factores que deben ser tomados en cuenta en el diseño de nuevos productos turísticos, elementos diversos que involucren no solo los intereses de la demanda, sino también los de la población residente. A su vez, esta oferta debe permitir el involucramiento y la participación deliberativa de la población. Es decir, deben ser actividades que generen una distribución justa de los beneficios, entre los visitantes y la población residente.

Sin duda, la economía del comportamiento representa una valiosa herramienta de política pública en diferentes áreas (Thaler y Sunstein, 2008), incluyendo al turismo; no obstante, es necesario desarrollar previamente los instrumentos que permitan diseñar las intervenciones y reconocer las señales, a la par de los empujones y otros heurísticos a los que se debe recurrir. En este sentido, es importante que los gobiernos de todos los niveles incorporen a sus administraciones unidades con esta perspectiva, que se ocupen de las políticas públicas, para incorporar las particularidades y necesidades de cada comunidad en la creación de dichas políticas y programas efectivos.

De tal manera, será posible generar pautas de desarrollo atendiendo aspectos más allá de los económicos, que permitan mover a la población de un status quo que en ocasiones es limitante, a un estado más deseable, en el que se generen beneficios tanto públicos como privados a bajo costo. Pequeños cambios en los programas generan cambios marginales en las preferencias. Como se observó en los resultados de la RCT, fue posible hacer cambios en las preferencias de los pobladores y visitantes, moviéndolos de su estado de conflicto y pasividad recurrente, a un momento que los llevó a la acción colectiva en beneficio propio y de su comunidad. De acuerdo con la revisión teórica y lo observado en este trabajo, las RCT pueden representar una herramienta para que los gobiernos asignen recursos económicos basados en la evidencia (Haynes et al., 2012), es decir, esta es una herramienta efectiva para la programación y la presupuestación.

En suma, los hallazgos encontrados permiten confirmar la importancia del diseño detallado de las intervenciones en grupos humanos, como medio para acercar los resultados a los propósitos de una intervención. Cuando los resultados de dicha intervención se corresponden con las expectativas ofertadas, el sesgo de retrospec- 
ción se reduce en un alto porcentaje. La aversión a las pérdidas es algo recurrente en las personas cuando deben decidir entre distintas opciones, así sea que la pérdida involucre exclusivamente al tiempo. Por último, se corrobora que el bienestar de las personas guarda relación con sus experiencias subjetivas y no solo cuando hay involucrados gastos económicos.

\section{Referencias}

Alcaldía de Tláhuac. (2020). Turismo local.http://www.tlahuac.cdmx.gob.mx/turismo -local/

Campos, R. M. (2017). Economía y psicología. Apuntes sobre economía conductual para entender problemas económicos actuales. El Colegio de México.

Cati, M. (2020). Behavioral tourism economics. SSRN. https://dx.doi.org/10.2139/ ssrn.3685059

Consejo Nacional de Evaluación de la Política de Desarrollo Social (Coneval). (2020). Índice de rezago social 2020. https://bit.ly/3CjV8go

Duflo, E. y Banerjee, A. (2011). Poor economics. A radical rethinking of the way to fight global poverty. Public Affairs.

Gursoy D., Hoc Nang Fong, L. y Sigala, M. (2020). Experimental research in tourism. Asia Pacific Journal of Tourism Research, 25, 707-709. https://doi.org/10.1080/ 10941665.2020.1775277

Haynes, L., Service, O., Goldacre, B. y Torgerson, D. (2012). Test, learn, adapt: developing public policy with randomised controlled trials. The Behavioural Insights Team. https://bit.ly/3fwZtTQ

Instituto Nacional de Estadística y Geografía (INEGI). (2020). Censo de población y vivienda 2020. https://bit.ly/3AldjuA

Instituto Nacional de Estadística y Geografía (INEGI). (2020). DENUE Directorio estadístico nacional de unidades económicas. https://bit.ly/37mXOeY

Jefatura de la Unidad Departamental (JUD) de Fomento a la Actividad Turística. (2018). Programa 2018. Alcaldía de Tláhuac.

Kahneman, D. y Tversky, A. (1979). Teoría prospectiva: un análisis de la decisión bajo riesgo. Econometrica, 47(2), 263-292. https://doi.org/10.2307/1914185 
Kahneman, D. (2013). Pensar rápido, pensar despacio. Penguin Random House y Grupo Editorial España.

Marín, L. (2020). Identidad y paisaje en el pueblo de San Nicolás Tetelco, Tláhuac y Milpa Alta, CDMX [investigación de licenciatura, Universidad Autónoma Metropolitana]. Repositorio institucional. https://bit.ly/3jquppN

Mendoza, A. (2019). Uso de la economía del comportamiento en la elaboración de políticas y programas para el fomento e impulso de actividades turísticas y recreativas. Estudio de caso San Nicolás Tetelco, Alcaldía Tláhuac de la Ciudad de México [tesis de maestría, Instituto Politécnico Nacional]. Repositorio Dspace. https://tesis.ipn.mx/handle/123456789/27807

Mosalev, A. I. (2020). Influence in the behavioral economy of the tourist industry. Advances in Economics, Business and Management Research, 128, 726-732. https://doi.org/10.2991/aebmr.k.200312.103

Norton, M., Mochon, D. y Ariely, D. (2011). The "IKEA effect": when labor leads to love. Revista de Psicología del Consumidor, 22(3), 453-460. https://doi.org/ 10.1016/j.jcps.2011.08.002

Ogaki, M. y Tanaka, S. (2017). Behavioral economics toward a new economics by integration with traditional economics. Springer Nature. https://bit.ly/3AdJAd4

Pfarr, N. (2016). Aplicando la economía del comportamiento al diseño. En E. Díaz y C. del Valle (Eds.), Guía de economía del comportamiento Vol. 1: políticas públicas (pp. 17-40). Instituto Mexicano de Economía del Comportamiento. https://bit.ly/2Vy1jNe

Richburg-Hayes, A. F., Anzelone, C., Dechausay, N., Datta, S., Fiorillo, A., Potok, L., Darling, M. y Balz, J. (2014). Behavioral economics and social policy: designing innovative solutions for programs supported by the administration for children and families. OPRE Report No. 2014-16a. Department of Health and Human Services. https://bit.ly/3jnejgN

Secretaría de Turismo (Sectur). (2013). Agendas de competitividad de los destinos turísticos de México. Ciudad de México (ACTCM 2013). https://bit.ly/3inHZLz

Simon, H. (1982). El comportamiento administrativo. Estudio de los procesos decisorios en la organización administrativa. Aguilar Argentina S.A. Ediciones.

Thaler, R. (2015). The making of behavioral economics misbehaving. W. W. Norton \& Company, Inc. 
Thaler, R. y Sunstein, C. (2008). Nudge. Improving decisions about health, wealth and happiness. Penguin Books.

Wallingre, N. (2014). Turismo, territorio y municipio. FACES, Revista de la Facultad de Ciencias Económicas y Sociales, 42-43, 143-164. https://bit.ly/2VuraWm

Wallstam, M., loannides, D. y Pettersson, R. (2020). Evaluating the social impacts of events: in search of unified indicators for effective policymaking. Journal of Policy Research in Tourism, Leisure and Events, 12(2), 122-141. https://doi.org/ 10.1080/19407963.2018.1515214 